\title{
Kinematics and Stellar Disk Modeling of Lenticular Galaxies
}

\author{
A. V. Zasov, ${ }^{1}$ A. V. Khoperskov, ${ }^{2}$ I. Yu. Katkov, ${ }^{1}$ V. L. Afanasiev, ${ }^{3}$ and S. S. Kaisin ${ }^{3}$ \\ ${ }^{1}$ Sternberg State Astronomical Institute, Moscow State University, Moscow, 119992 Russia \\ ${ }^{2}$ Volgograd State University, Volgograd, 400062 Russia \\ ${ }^{3}$ Special Astrophysical Observatory of the Russian AS, Nizhnij Arkhyz 369167, Russia
}

(Received February 17, 2012; Revised July 2, 2012)

\begin{abstract}
We present the results of spectroscopic observations of three S0-Sa galaxies: NGC 338, NGC 3245, and NGC 5440 at the SAO RAS 6-m BTA telescope. The radial distributions of the line-of-sight velocities and radial velocity dispersions of stars and ionized gas were obtained, and rotation curves of galaxies were computed. We construct the numerical dynamic $N$-body galaxy models with $N \geq 10^{6}$ point masses. The models include three components: a "live" bulge, a collisionless disk, dynamically evolving to the marginally stable state, and a pseudo-isothermal dark halo. The estimates of radial velocities and velocity dispersions of stars obtained from observations are compared with model estimates, projected onto the line of sight. We show that the disks of NGC 5440 and the outer regions of NGC 338 are dynamically overheated. Taking into account the previously obtained observations, we conclude that the dynamic heating of the disk is present in a large number of early-type disk galaxies, and it seems to ensue from the external effects. The estimates of the disk mass and relative mass of the dark halo are given for seven galaxies, observed at the BTA.
\end{abstract}

\section{INTRODUCTION}

Lenticular galaxies (S0) are disk systems which generally differ from spiral galaxies by a very small amount of neutral gas and young stars in the disk. As a consequence, they do not have any contrast spiral arms or vigorous centers of star formation. These galaxies exist not only in clusters and groups of galaxies, but also among the field galaxies. Alike the spiral galaxies, the luminosity ratio of disk and bulge in lenticular galaxies varies in a broad range of values, although on the average it is higher. In most cases, similar to the spirals, the bulges here are actually the pseudo-bulges [1]. The surface brightnesses of the disks of S0 galaxies are not lower but rather

higher than those in the spiral galaxies of similar luminosity [2, 3]. At the same time, there is no abrupt transition from the S0 galaxies to the later types (S0/a, Sa).

The question about the features of S0 galaxies and their evolutionary relationship with the spiral galaxies is actively discussed in the literature (see [3-10] and references therein). Various authors have considered several different scenarios of the transformation of a galaxy with an initially intensive 
star formation in the disk into a lenticular galaxy. They may be linked both with the internal causes (high efficiency of star formation leading to the depletion of gas, the sweeping-out of gas from the disk at the stage of active nucleus or a burst of star formation) and external (heating up or sweeping-out of gas by the interaction with the intergalactic medium, an cessation of accretion into the disk, merging of small galaxies, followed by a burst of star formation, tidal interactions with nearby galaxies or a massive cluster of galaxies). Merging with smaller systems and strong tidal perturbations, even if they occurred in the distant past, leave their nonvanishing trace, dynamically heating the stellar disk $[6,11]$. At the same time though, the sweeping-out of gas or its rapid depletion at the epoch when the disk has already been mostly formed, would not significantly affect its dynamics.

The observations show that lenticular galaxies significantly differ not only by the shapes of their rotation curves, but also by the radial profiles of velocity dispersion of their stellar components. Velocity dispersion, measured along the line of sight, is typically decreasing monotonously with distance from the center. But in some cases, even at a distance of two radial scales from the center of the disk $(r=2 h)$, the radial component of dispersion would set at a level of one-half of the rotation velocity (the corresponding estimates, based on the published data are given in Table 1 from Zasov et al. [12]). In other cases, the velocity dispersion turns out to be the same as for most of spiral galaxies. The kinematic models show that the kinematics of the disks of some lenticular galaxies does not demonstrate any anomalies. As an example, note the measurements of the kinematics of the planetary nebulae in the disk of NGC 1023 by Cortesi et al. [13]. In such cases, we can conclude that the evolution of a given lenticular galaxy has progressed in a relaxed manner, without any considerable external perturbations.

Obviously, neither the magnitude of the velocity dispersion, nor its relation to the rotation velocity would already imply whether the galaxy has experienced a strong heatup of the dynamic disk, or the velocity dispersion of the disk stars is close to the minimum (marginal) level, ensuring its dynamic stability. The disk can approach the state of marginal stability via internal processes: dynamic heatup, related to the development of local instabilities both in the disk plane, and in the perpendicular direction (bending instability) [14, 15]. At the presence of a massive spherical component (a halo, bulge), the velocity dispersion of stars in the marginally stable disk will be much lower than the circular velocity. However, as shown by numerical models, it reaches $30-50 \%$ of circular velocity without any external effects if the disk is self-gravitating, i.e. the mass of spheroidal components in whose field the disk is embedded,is relatively small (see, for example, [16, 17]). Note that a rough mass estimate of the disks of spiral galaxies, assuming their marginal stability at two 
radial scales from the center $(r \approx 2 h)$ is statistically consistent with the photometric estimates of the disk mass [12], although for the individual galaxies the differences may be quite large.

In general, the disk of the galaxy at any distance from the center can have an excessive (for stability) velocity dispersion. Below we consider the disk to be dynamically overheated if at a sufficient range of distances from the center the observed velocity dispersion of the stellar component $C_{\text {obs }}$ is systematically higher than the dispersion estimate for the marginally stable disk by a value not exceeding the characteristic scatter of points along the radial profile of $C_{\text {obs }}(r)$ (typically-10$20 \mathrm{~km} / \mathrm{s}$ ). However, even in the case of a dynamical overheating of the disk, it still makes sense to build a model of the galaxy under the assumption of marginal stability of its stellar disk: a comparison of this model with observations provides a constraint on the commonly used maximum disk model, containing the "highest possible" density of the disk which matches with the observed rotation curve. This model, if it takes into account not only the shape of the rotation curve, but also the radial profile of the velocity dispersion of the old stellar population of the disk, making up its bulk, may be called the refined maximum disk model. The examples of this approach to estimate the disk mass or the disk mass-to-luminosity ratio are discussed, e.g., by Bottema [18, 19], Zasov et al. [12, 17, 20], and with regards to the LSB galaxies-by Saburova [21]. If the actual disks of galaxies have smaller masses than the mass, ensuing from the marginally stable disk model, or if the observed velocity dispersion of the disk stars is higher than the model velocity dispersion projected onto the line of sight, this argues for the dynamic overheating of the disk.

Note that the stability of the disk depends on a number of factors difficult to account for. The analytical expressions for the stability criterion are obtained only in the local approximation, and only given the essentially simplifying assumptions. More reliable results may be achieved by constructing the dynamically stable numerical $N$-body models, satisfying the observed distributions of brightness and rotation velocity, and comparing the "model" velocity dispersion profiles with observations. Numerical models are particularly important for the galaxies with high velocity dispersions of the disk stars, for which the rotation curve of gas is absent. In this case, the observed rotation curve of stars has to be corrected for the asymmetric drift, and this problem has an analytical solution only in the case of a minor dispersion, when the ratio of squares of the stellar velocity dispersion to circular velocity is much smaller than unity.

Construction of $N$-body models for three S0 galaxies (NGC 1167, NGC 4150, NGC 6340) and an SBa-type galaxy (NGC 2273) from the BTA observations has been previously implemented in Zasov et al. [22]. Three-dimensional numerical dynamical galaxy models were constructed with the lowest possible velocity dispersion of disk stars which provides the disk stability. The models included 
a stellar disk, which is close to the maximum disk, a bulge with an initial density distribution approximated by the King's law, and a pseudo-isothermal halo. The spectroscopic measurements of NGC 6340 were later reprocessed and refined by Chilingaryan et al. [23], reconfirming the previous findings. A comparison of models with the observational data has shown that only one galaxy out of the four modelled, the SBa galaxy NGC 2273 demonstrates an agreement of the observational data with the assumption that the stellar disk is close to the marginally stable state. In this respect this galaxy is similar to many spiral galaxies of later types, in which the stellar velocity dispersion is close to the stability threshold, at least at distances of about two radial scales of the disk from the center[12].

In this paper we present the results of spectroscopic observations of three early-type galaxies: NGC 338, NGC 3245 and NGC 5440 conducted at the 6-m BTA telescope of the Special Astrophysical Observatory of the Russian Academy of Sciences (SAO RAS), and compare the obtained velocity measurements with the numerical models of galaxies with marginally stable disks. Their images from the SDSS survey are presented in Fig. 1. The use of two spectral cuts made it possible to check whether we were right in our choice of the position angle $\mathrm{PA}_{0}$ of the dynamical major axis.
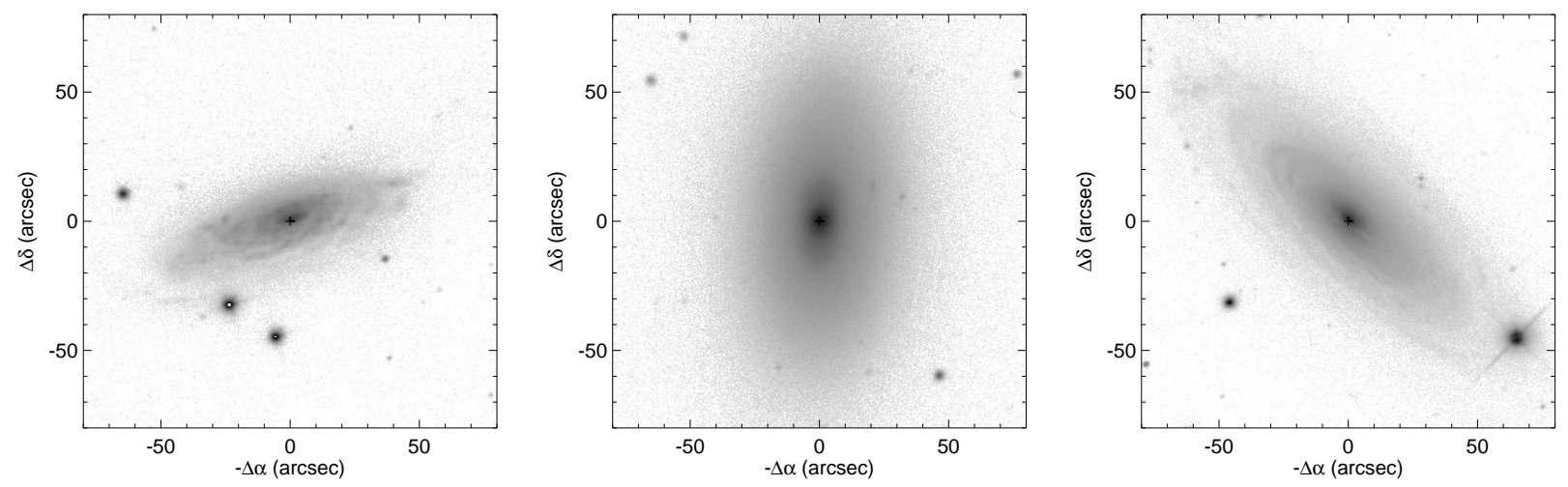

Figure 1. The SDSS survey images of the NGC 338, NGC 3245 and NGC 5440 galaxies in the $g$ filter.

In principle, spectral cuts along the major and minor axes of galaxies also allow to obtain from observations the ratio of velocity dispersions along different axes. However, for the galaxies we consider in this paper these estimates appeared to be unreliable: the results came out to be very sensitive to the choice of law, approximating the observed velocity dispersion profiles along the major and minor axes, and the rotation velocity of the stellar component. Minor variations in the source data approximation (within the error of observations) significantly affect the resulting estimates of the velocity dispersion ratio $C_{z} / C_{r}$ in the vertical and radial directions, and hence we 
do not give them in this paper.

\section{DESCRIPTION OF GALAXIES}

NGC 338. This is a type Sa or Sab galaxy with weak spiral arms, a member of a low-density group. In the SDSS and 2MASS survey images the galaxy looks like an early-type disk galaxy with a bright nucleus, extended bulge and a disk, strongly inclined to the line of sight (Fig. 1). The SDSS images reveal in the disk an indistinctly blurred spiral structure with separate star forming regions, notable for their blue color. The galaxy contains a relatively large (for lenticular galaxies) amount of H I [24], so it can be regarded as an early-type spiral galaxy with weak star formation. The photometry and kinematics of this galaxy was previously considered in $[1,25]$. The disk has a relatively low central (deprojected) brightness and a large radial scale in the photometric $R$-band (about $5.8 \mathrm{kpc}$ at a distance of $65.1 \mathrm{Mpc}$ ) and is significantly inferior to the bulge in luminosity. However, as noted by the cited authors, the conclusion about the anomalously bright and extended bulge (the effective radius of $r_{e} \approx 4.7 \mathrm{kpc}$ ) may be due to the error of the photometric decomposition of brightness into the components within the "exponential disk + Sérsic bulge" model, and to the possible presence of dust in the center of the galaxy.

The rotation curve of the galaxy reaches the plateau quite fast, within a few kpc from the center [25], the maximum velocity of rotation is estimated to be $300 \pm 16 \mathrm{~km} / \mathrm{s}$ [26]. However, a large-scale asymmetry of the velocity field, possibly related to the interaction with a neighboring galaxy, has prevented the authors of [25] from using the obtained velocity estimates in the galaxy modeling. Following their work, we adopted the position angle of major axis $\mathrm{PA}_{0}=108^{\circ}$ and the angle of inclination of the disk $i=64^{\circ}$. Using the spectral cuts we have constructed rotation curves both for the gas and for the stellar disk, up to a distance of approximately $50^{\prime \prime}$ from the center.

NGC 3245. This is a single lenticular galaxy, possessing a bright central disk (a lens) with a sharp outer boundary at a distance of $r \approx 15^{\prime \prime}-20^{\prime \prime}$ and an external main disk. The galaxy has a very high gradient of the line-of-sight velocity within $4^{\prime \prime}$ from the center [27]. There are no traces of spiral structure, H II zones or star-forming regions, although we managed to detect some faint emissions in the spectrum of the central disk, indicating a rapid rotation within $10^{\prime \prime}$. The galaxy has a Seyfert nucleus of a low-level activity. According to the photometric measurements [28] the galaxy has a compact bulge with an effective radius of $r_{e}=3^{\prime \prime} 3$ and a disk with a radial scale of $h=17^{\prime \prime} 6$, or $1.5 \mathrm{kpc}$, given the adopted distance of $18 \mathrm{Mpc}$. The kinematics of the galactic disk was studied in [29], but the measurements cover only the central $17^{\prime \prime}$, where the angular velocity 
Table 1. The log of observations

\begin{tabular}{c|c|c|c|c}
\hline $\begin{array}{c}\text { Galaxy } \\
\text { name }\end{array}$ & $\begin{array}{c}\text { Date of } \\
\text { observations }\end{array}$ & $\begin{array}{c}\text { Slit orientation } \\
\text { PA, deg }\end{array}$ & $\begin{array}{c}\text { Exposure, } \\
\text { s }\end{array}$ & $\begin{array}{c}\text { Seeing, } \\
\text { arcsec }\end{array}$ \\
\hline NGC 338 & Oct 2, 2006 & 289 & $7 \times 1200$ & 2.7 \\
& Sep 18, 2007 & 197 & $9 \times 1200$ & 2.4 \\
NGC 3245 & Apr 14, 2007 & 355 & $6 \times 1200$ & 4.0 \\
& Apr 7, 2008 & 267 & $6 \times 1200$ & 3.3 \\
NGC 5440 & May 12, 2007 & 230 & $8 \times 1200$ & 1.3 \\
& May 16, 2007 & 320 & $2.7 \times 1200$ & 1.5 \\
\hline
\end{tabular}

is nearly constant. From the obtained spectral cuts we have constructed the stellar disk rotation curve, slowly receding at $r>30^{\prime \prime}$. We used the photometrically determined orientation angles of the disk.

NGC 5440. This poorly studied lenticular galaxy is a possible member of a diffuse group. The bright inner region of the galaxy passes into a disk of low surface brightness, in which the low contrast arms or ring fragments are visible. The results of photometry of the galaxy, and two-dimensional photometric decomposition into the bulge and disk are given in [28]. The effective radius of the bulge and radial scale of the disk amount to $33^{\prime \prime} 4$ and $155^{\prime \prime} 7$, respectively, which corresponds to $4.0 \mathrm{kpc}$ at a distance of $52 \mathrm{Mpc}$. The position angle of the photometric major axis is $\mathrm{PA}_{0}=46^{\circ}$, which is consistent with our measurements with two slit orientations.

\section{OBSERVATIONS AND DATA REDUCTION}

The observations of galaxies were carried out in the primary focus of the 6-m BTA telescope of the Special Astrophysical Observatory (SAO RAS) over 2006-2007 using the SCORPIO focal reducer [30] in the long-slit mode (with the slit size of $6^{\prime} \times 1^{\prime \prime}$ ). We used the VPHG 2300G grism with the operating spectral range of 4800-5550 $\mathrm{A}$, which contains the absorption lines of $\mathrm{Fe}, \mathrm{Mg}$, Ti, etc., as well as the $\mathrm{H} \beta$, [O III], [N I] emissions. The EEV 42-40 detector with the CCD sized $2 \mathrm{~K} \times 2 \mathrm{~K}$ in the $1 \times 2$ binning mode provided the $0.35^{\prime \prime} / \mathrm{px}$ scale along the slit. The orientation of the slit, the total exposures and atmospheric conditions are given in the log of observations (Table 1). The characteristic resolution of the spectra obtained is $2.6 \AA$, which corresponds to $65 \mathrm{~km} / \mathrm{s}$ in terms of velocity.

Initial data reduction included standard steps: subtracting the averaged bias frame, accounting for the inhomogeneous illumination and CCD sensitivity variations using the frames of the continu- 
ous spectrum calibration lamp (flat field), removing the traces of cosmic ray particles, construction of a two-dimensional dispersion equation from the spectrum of a He-Ne-Ar calibration lamp, spectrum linearization, summation of the spectra, subtracting the spectrum of the night sky taking into account the instrumental profile variations along the slit (for the details of sky subtraction procedure, see [31]). The dispersion equation was parameterized by a polynomial surface with the fifth- and fourth-degree polynomials along and across dispersion, respectively. The typical error of the dispersion equation is $0.03 \AA$ for an individual spectral line. The transition to absolute fluxes was not performed. At each stage of the initial processing the images with Poisson flux errors were calculated.

For the further reduction of stellar spectra the ULySS software package [32, 33] was used, adapted to the SCORPIO data. ULySS was primarily applied to determine the shape of the spectrograph instrumental profile and its variations in the frame. For this purpose we obtained the frames of the dawn (or dusk) sky, which were taken the same night as the objects. To determine the variation over the field, the spectrum of the dawn sky was split into 60 elements along the slit and averaged for each element. The spectrum was divided into seven overlapping segments by wavelength. After that, each part of the spectrum was approximated by the high resolution solar spectrum $(\lambda / \Delta \lambda \approx 10000)$, convolved with the instrumental profile in the form of the Gauss-Hermite function with the $v, \sigma, h 3$ and $h 4$ parameters [34]. The instrumental profile was used for the night sky spectrum subtraction procedure and in the analysis of the spectra of galaxies.

As the next step, the ULySS software package was used for the pixel by pixel approximation of the observed spectra by the model spectra of stellar populations by minimizing the $\chi^{2}$ residual. The object spectrum was adaptively binned in advance. The core of this procedure consists in dividing the spectrum into the elements along the slit, based on the condition that the signal-to-noise ratio in each averaged element would be not less than the preassigned value (typically 20-50).

Modeling the spectra, high-resolution PEGASE.HR models [35] were used for the Simple Stellar Population (SSP) with star formation history, described by a single burst and the Salpeter Initial Mass Function. Before fitting, the PEGASE.HR model grid was subjected to the convolution with the instrumental profile for the given slit position (for the given bin). As the next step, within the fitting algorithm the selection of the stellar population spectrum from the model grid was made for the current parameters of stellar age $T$ and metallicity $[\mathrm{Fe} / \mathrm{H}]$. This was followed by the convolution with the Line-Of-Sight Velocity Distribution function (LOSVD), which was taken in the form of a Gauss-Hermite function. After that, the spectrum was multiplied by the polynomial continuum, which allows to formally take into account internal absorption in the galaxy, as well 
as the unaccounted spectral sensitivity curve of the CCD detector. After fitting the spectrum for each bin, we obtained the estimates of the line-of-sight velocity $v_{r}(\mathrm{~km} / \mathrm{s})$, stellar velocity dispersion $C_{\text {obs }}(\mathrm{km} / \mathrm{s})$, the parameters characterizing non-Gaussianity of LOSVD: $h 3$, $h 4$, as well as the SSP-equivalent estimates of the age $T$ (Gyr) and metallicity $[\mathrm{Fe} / \mathrm{H}](\mathrm{dex})$. In this paper we used solely the estimates of the velocity and velocity dispersion. A more detailed description of the fitting procedures and discussion of the parameter degeneracy and algorithm stability, see the papers $[32,36,37]$.

\section{PRINCIPLES OF MODELING}

The basic principles of constructing the numerical equilibrium galaxy models are presented in [22] and in the monograph by Fridman and Khoperskov, titled the Physics of Galactic Disks [14]. The basis of dynamic galaxy models is the numerical integration of the equations of motion of the $N$ bodies, where $N$ is a few million (depending on the model) particles, imitating the disk, bulge and the dark halo. The solution of Poisson's equation for a given distribution of particles was found at each integration step with the use of the TreeCode algorithm.

We assumed that the gas rotation curve is close to the curve of circular rotation, and the rotation velocity, determined by the stars is lower than circular by the value of asymmetric drift. The construction of galaxy models, in which the disks are located at the border of gravitational stability was done via the method of successive approximations by a gradual step-by-step increase of the velocity dispersion of slightly unstable disk to obtain a dynamically stable state, preserved during several revolutions of the peripheral regions of the disk. The estimates of line-of-sight velocities of stars and gas parallel with the stellar velocity dispersions, obtained from the observations, were compared with their model profiles, calculated along the major axis and projected onto the line of sight.

To compare the observed radial profile of line-of-sight velocity dispersion with the model profile we took into account the presence of the bulge: a relative contribution of the disk and bulge to the spectrum was considered to be proportional to their line-of-sight column density at a given radial distance. The radial scale of the disk mass distribution in all the models was assumed to be equal to the photometrically determined brightness scale, while the mass-to-luminosity ratio was considered as a free parameter. The masses of the disk and other components were determined so that the radial profile of the stellar disk line-of-sight velocity, calculated for a given model, would be as close as possible to the observed stellar velocity profile (i.e., not corrected for the asymmetric drift), while 
Table 2. Integral parameters of disks: results of modeling

\begin{tabular}{c|l|c|c|c}
\hline $\begin{array}{c}\text { Galaxy } \\
\text { name }\end{array}$ & Type & $\begin{array}{c}\text { Disk mass } \\
M_{d}, 10^{10} M_{\odot}\end{array}$ & $M_{h} / M_{d+b}$ & $\begin{array}{c}\text { Disk } \\
\text { state }\end{array}$ \\
\hline NGC 1167 & $\mathrm{S} 0$ & 39 & 0.51 & $\mathrm{o}$ \\
NGC 2273 & $\mathrm{SBa}$ & 8.7 & 0.65 & $\mathrm{~m}$ \\
NGC 4150 & $\mathrm{S} 0$ & 0.53 & 0.56 & $\mathrm{o}$ \\
NGC 6340 & $\mathrm{S} 0$ & 4.54 & 0.66 & $\mathrm{o}$ \\
\hline NGC 338 & $\mathrm{Sa}$ & 15.4 & 0.32 & $\mathrm{~m}$ \\
NGC 3245 & $\mathrm{S} 0$ & $1.1:$ & $0.6:$ & $\mathrm{m}$ \\
NGC 5440 & $\mathrm{Sa}$ & 19.3 & 0.56 & $\mathrm{o}$ \\
\hline
\end{tabular}

the circular rotation curve would agree with the velocity estimates, found from the emission lines, if present. In contrast to the previous work [22], the $N$-body model considered the bulge to be "live", and its parameters were determined from the condition of the compliance of the model velocity dispersion of the "disk + bulge" system with the observed value for the central part of the galaxy.

\section{RESULTS OF KINEMATIC ESTIMATES, COMPARISON WITH MODELS}
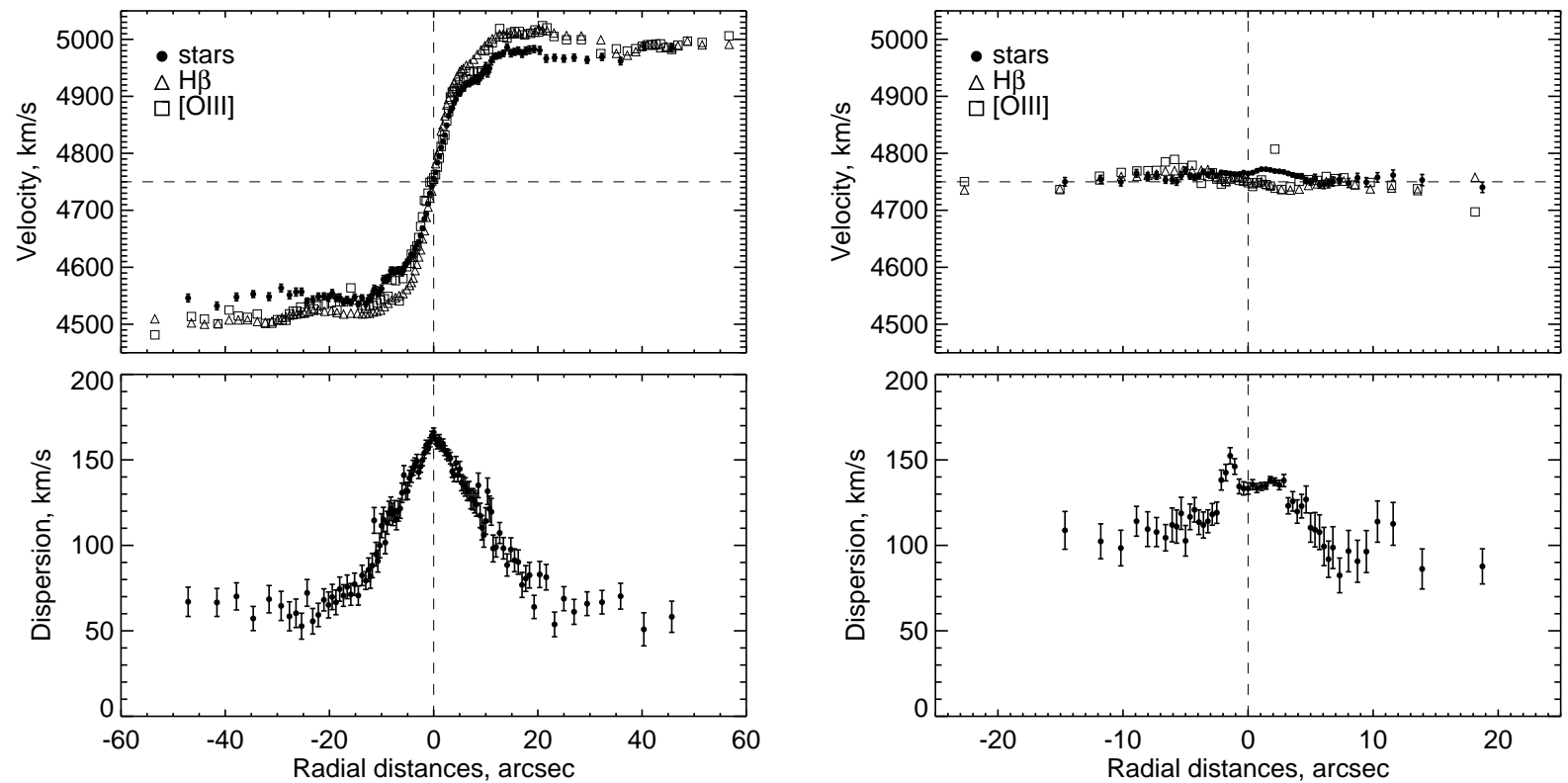

Figure 2. The NGC 338 galaxy. Left: results of measurements along the major axis, right: measurements along the minor axis. Top: radial profiles of line-of-sight velocities of stars and ionized gas, bottom: stellar velocity dispersion. The error bars of line-of-sight velocities of ionized gas are not shown because their size is not larger than the size of icons. 

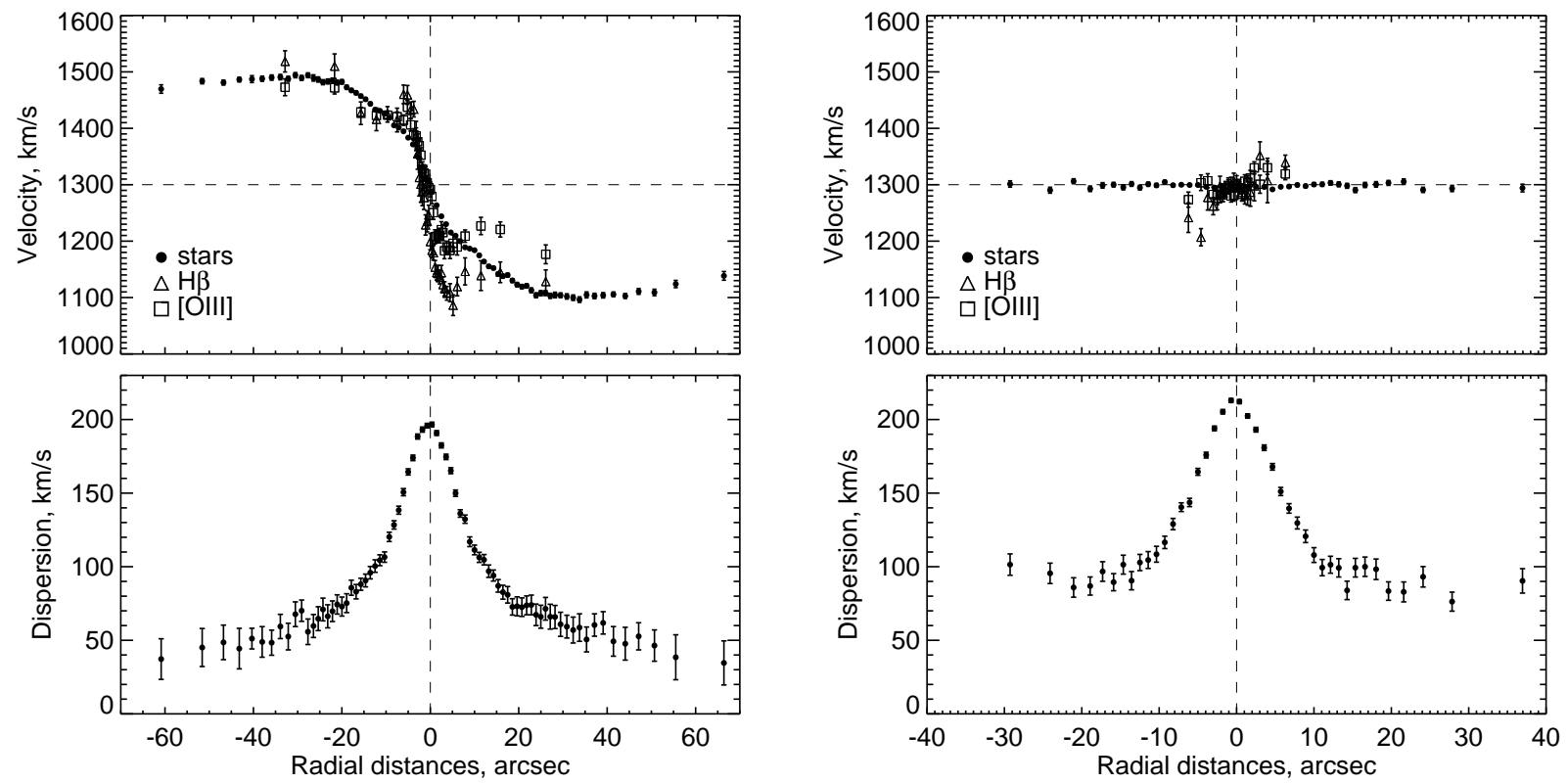

Figure 3. The same as in Fig. 2, but for the NGC 3245 galaxy.
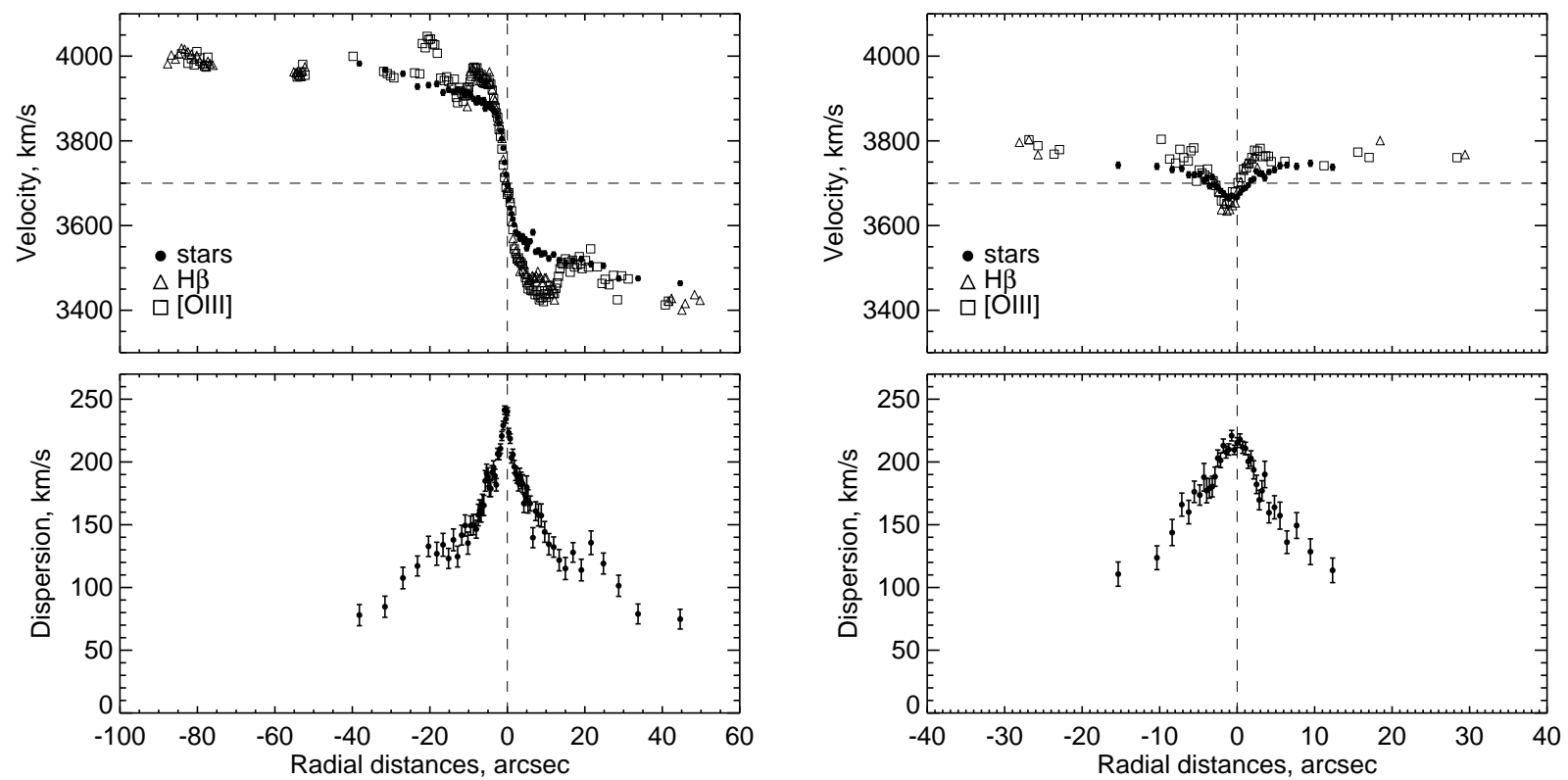

Figure 4. The same as in Fig. 2, but for the NGC 5440 galaxy.

The obtained radial profiles of the line-of-sight velocities and stellar velocity dispersions are demonstrated in Figs. 2-4. The reconstructed rotation curve for a marginally stable disk of each galaxy (i.e. for a stable disk model with the maximal density possible), as well as the model and observed radial profiles of stellar velocity dispersions are shown in Fig. 5. The resulting estimates of the parameters of galaxy components are given in Table 2. The photometric parameters of 

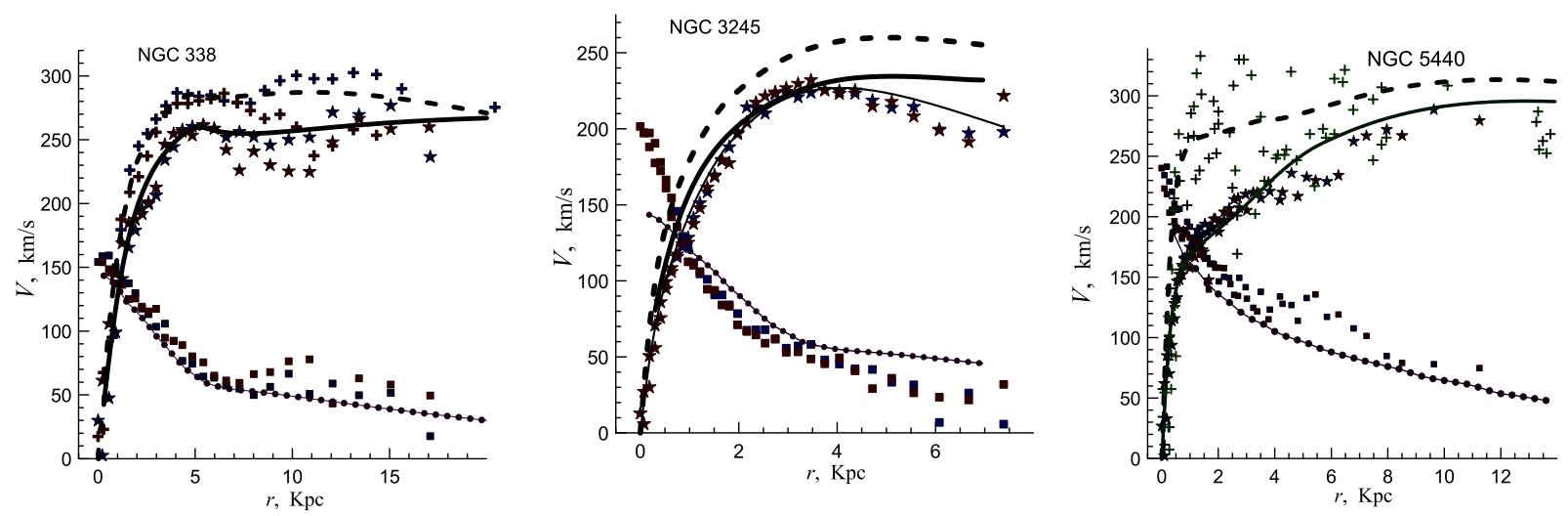

Figure 5. The results of dynamic modeling. The thick dashed line describes the circular rotation velocity,

the thick solid line-stellar rotation velocity in the model, the stars-stellar rotation velocity from observations, crosses-gas rotation velocity from observations, squares-stellar velocity dispersions from observations, the thin line with small circles-velocity dispersion along the line of sight in the marginally stable disk model. For NGC 3245 two model stellar rotation curves are given (the thin and thick lines), corresponding to different velocity dispersions along the $z$-coordinate (see text).

galaxies, if their source is not indicated, are taken from the NED database ${ }^{1}$. The three-dimensional axisymmetric model includes a truncated King's bulge with a fixed total mass $M_{b}$, an exponential disk and a spherical pseudo-isothermal halo. The solid and dashed lines in Fig. 5 describe the model curves of circular velocity and stellar disk velocity, respectively. The descending solid line in the bottom part of the figure demonstrates the radial profile of the stellar disk velocity dispersion projected onto the line of sight for the model constructed. Although all the three galaxies belong to the early morphological types, their rotation curves turned out to be different in shape, indicating different mass distributions in galaxies. The NGC 338 galaxy reveals the rotation curve which comes to a plateau at $r \approx 3 \mathrm{kpc}$, and displays noncircular velocities at large $r$. In NGC 3245 the rotation curve for the stars passes through a maximum, while in NGC 5440 the curve is notable for a very high velocity gradient in the central region, which gradually decreases with distance from the center.

The "live" bulge was built from dynamic particles with the same masses as for the disk. In an iterative process of the construction of an equilibrium marginally stable disk, the parameters, describing the density distribution in the bulge remained the same, but the bulge velocity dispersion slightly varied and its flattening slightly increased due to the gravitational effects that the disk imposes on the bulge. During the galaxy modeling, a slight redistribution of matter took place in the bulge, especially in the centermost region, where the density increased. However these changes

\footnotetext{
${ }^{1}$ http://ned.ipac.caltech.edu/
} 
Table 3. Bulge parameters

\begin{tabular}{c|c|c|c}
\hline $\begin{array}{c}\text { Galaxy } \\
\text { name }\end{array}$ & $\begin{array}{c}\text { Bulge mass } \\
M_{b}, 10^{10} M_{\odot}\end{array}$ & $\begin{array}{c}\text { Scale } \\
r_{b}, \mathrm{kpc}\end{array}$ & $\begin{array}{c}\text { Radius } \\
r_{b}^{\max }, \mathrm{kpc}\end{array}$ \\
\hline NGC 338 & 4.80 & 1.12 & 6 \\
NGC 3245 & 0.45 & 0.35 & 1.6 \\
NGC 5440 & 3.20 & 0.38 & 3.6 \\
\hline
\end{tabular}

did not have a significant impact on the dynamics of the disk. The parameters of the bulges of galaxies from the models constructed (the mass, scale, and the radius of the bulge in King's model) are listed in Table 3.

Let us give comments on each galaxy.

$N G C 338$. This is a fast rotating galaxy, its circular velocity reaches $280-300 \mathrm{~km} / \mathrm{s}$. The profile of line-of-sight velocities was obtained both from the absorption lines, and emissions in $\mathrm{H} \beta$, [NI] and [O III] (Fig. 2). The observations of the galaxy were carried out with the slit orientations $17^{\circ}$ and $108^{\circ}$ (the latter angle is close to the major axis position angle). The rotation curve reaches saturation at $r \approx 4 \mathrm{kpc}\left(12^{\prime \prime}\right)$, however at $r>8 \mathrm{kpc}\left(25^{\prime \prime}\right)$ rotation velocities at the opposite sides from the center differ. This is especially noticeable in the curves along the major axis, where the velocity difference (taking into account the projection effects) reaches $50 \mathrm{~km} / \mathrm{s}$. This confirms the asymmetry of the velocity field, earlier found by Noordermeer and van der Hulst [25], being the most prominent in the H I line. Our measurements show that this anomaly affects not only the gas, but the stellar component also. At the same time, the decrease in the rotation velocity of stars is compensated by increased velocity dispersion, indicating that this anomaly does really exist. One may propose that its reason is due to the perturbation of the velocity field of stars and gas in the process of merger of a small satellite galaxy. However without the data on the velocity field of the stellar disk we can not perceive whether this effect is local or the anomaly covers a larger area of the disk.

The velocity of rotation of the gas, determined from the emission lines, markedly exceeds the velocity of rotation of the stellar disk, which may be explained, both qualitatively and quantitatively, by a higher stellar velocity dispersion. The observed velocity dispersion, which is particularly high within the $r \leq 15^{\prime \prime} \approx 5 \mathrm{kpc}$ region, dominated by the galactic bulge, rapidly decreases and reaches a plateau at $r \approx 20^{\prime \prime}$. Along the minor axis $\left(\mathrm{PA}=17^{\circ}\right)$ the velocity dispersion profile can be traced more poorly. The dispersion remains high up to $r \approx 10^{\prime \prime}-15^{\prime \prime}$, being determined mainly by the contribution of the bulge. 
A comparison of the stellar line-of-sight velocity dispersion profile, obtained from observations (squares) with that calculated for the marginally stable disk model (the bottom curve in Fig. 5, left) shows that the observed velocity dispersion varies along the radius, following the model profile, and only slightly (on the average by $10-20 \mathrm{~km} / \mathrm{s}$ ) exceeds the model estimates. The typical dispersion errors have roughly the same value. We can therefore conclude that the disk within the radius of $7-8 \mathrm{kpc}\left(22^{\prime \prime}-25^{\prime \prime}\right)$ is close to the marginally stable state, and hence was not subjected to a strong dynamic heating. At larger distances, the majority of velocity dispersion estimates lie higher than the model dependency, which suggests a noticeable overheating of the disk.

NGC 3245. The galaxy turned out to be very difficult to simulate. Moreover, we were unable to develop an adequate self-consistent model that would properly describe the kinematics of the entire disk: the external and internal regions of the disk deviate from the model of the maximal marginally stable disk, though for different reasons, and therefore require separate discussion. Let us consider this galaxy in more details.

The profiles of the rotation velocity and velocity dispersion of the galaxy have a symmetrical form. However, the galaxy has a number of dynamic features. Firstly, within the radius $10^{\prime \prime}-15^{\prime \prime}$ $(\approx 1 \mathrm{kpc})$ from the center the rotation velocity of the gas (projected onto the line of sight) exceeds the velocity of the stellar disk. It allows to assume that the gas forms a dynamically isolated disk, inclined with respect to the stellar disk, since a cut along the minor axis of the galaxy exhibits a non-zero gradient of the gas velocity (Fig. 3). It is impossible to get a reliable estimate of this gradient from the data available, since the emissions are observed only near the nucleus.

Another feature of this galaxy is a peak revealed on the stellar disk rotation curve: starting at $r \approx 4 \mathrm{kpc}$ (about $45^{\prime \prime}$ ) the velocity of its rotation is declining. The velocity dispersion ratio $C_{z} / C_{r} \approx 0.5-0.7$, typical of galactic disks (in agreement with numerical models which are stable with respect to the bending perturbations) does not allow to reproduce the observed peak of rotation. To explain the shape of this curve, we have to assume that the region of the peak and the subsequent rotation velocity decline satisfies the condition $C_{z} \approx C_{r}$, i.e., the disk has a relatively large vertical velocity dispersion, which makes the thickness of the stellar disk to be very large (a scaleheight is about $1.2 \mathrm{kpc}$ near the peak of the curve, which approximately corresponds to the height of the thick disk of our Galaxy). Moreover, the vertical scale of the stellar disk should further increase with radius as a result of the surface density decrease in the disk. The stellar disk rotation curve, corresponding to this model, is shown by a continuous thin line in Fig. 5(middle). However, the circular velocity curve of the galaxy (dashed line in Fig. 5) may have no peak, and apparently reaches a plateau. 


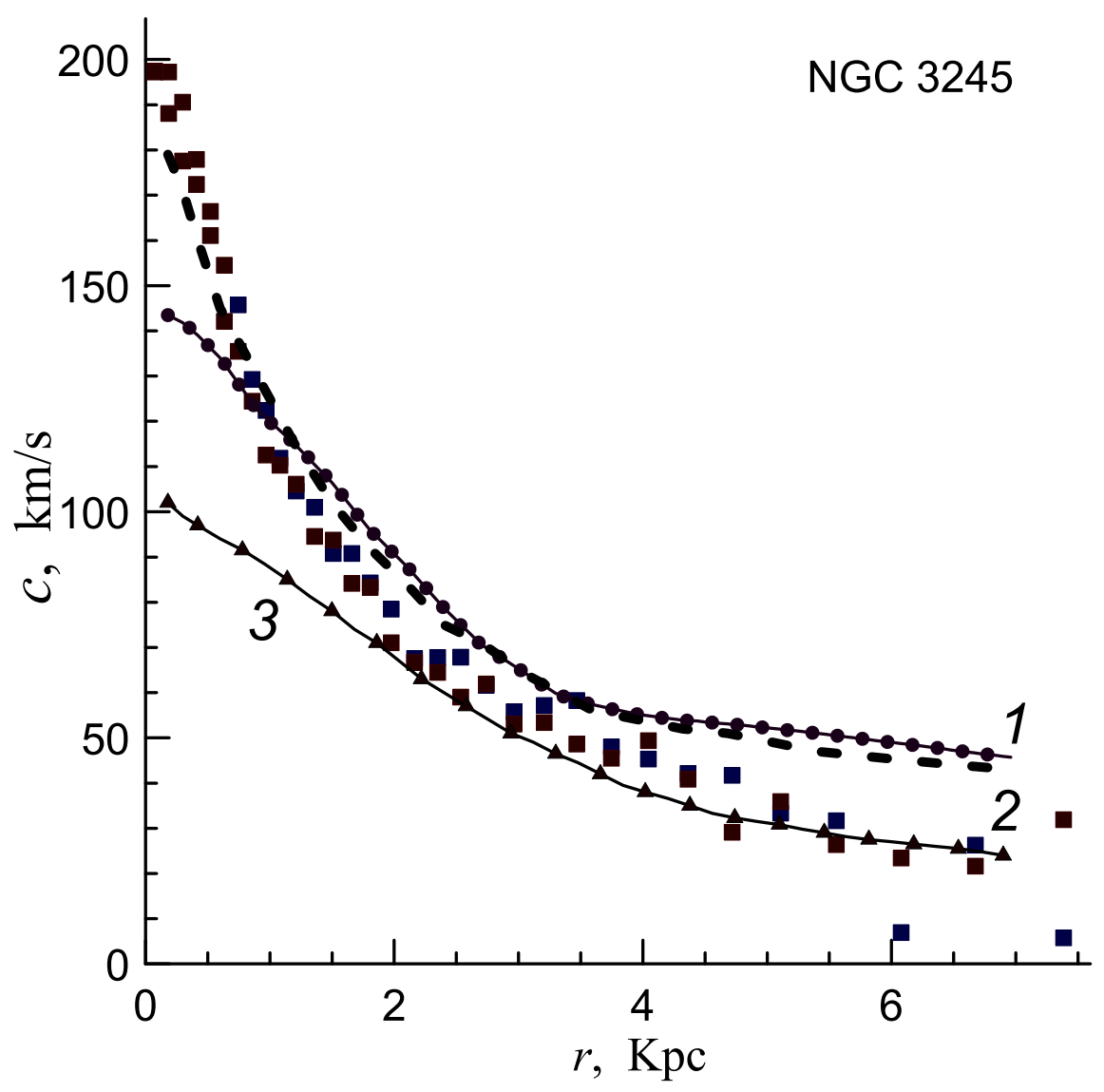

Figure 6. The radial distributions of the velocity dispersion along the line of sight for the NGC 3245 galaxy. The observations are marked by squares, curve (1) corresponds to the model shown in Fig. 5, the dashed line (2) - model with an overheated central part of the disk, (3) - model with a massive halo and a lightweight, marginally stable disk.

The third feature of the galaxy is high velocity dispersion of stars in the central region, not simulated within a simple model with the lowest possible velocity dispersion. The observed velocity dispersion of disk stars begins to increase towards the center-even before reaching the area, dominated by the bulge. In Fig. 6, in addition to the model of maximal marginally stable disk (curve (1)), a dashed line (2) shows the results of the model, where the disk density is lower (that is the radial velocity dispersion exceeds the minimum value, required for disk stability), but the central part of the disk is overheated. This inner extended region of the disk with high stellar velocity dispersion can be connected with the central thickening of the disk (a lens, a pseudo-bulge) of this galaxy.

Note, that out of all the galaxies we examined, NGC 3245 is the only one for which the refined maximal disk model predicts over a large distance interval a higher than observed stellar velocity 
dispersion: by $10-20 \mathrm{~km} / \mathrm{s}$ for $r=1-4 \mathrm{kpc}$ (around $10^{\prime \prime}-45^{\prime \prime}$ ) and by $25-30 \mathrm{~km} / \mathrm{s}$ for $r=6 \mathrm{kpc}$ (around $70^{\prime \prime}$ ) (see Fig. 5). Since the velocity dispersion of a marginally stable disk is minimally acceptable, we must conclude that in this case, the model of a maximal disk overestimates its density. Outside $r=2 \mathrm{kpc}$ (around 20") the best agreement between the observed velocity dispersion with that expected for a marginally stable disk (curve (3) in Fig. 6) is achieved at the total mass of the disk of $M_{d}=1.1 \times 10^{10} M_{\odot}$, what is roughly twice smaller than for the maximal disk.

Thus, the dynamic features of the observed galaxy can be explained if we accept that its disk is "overheated" in the central part, whereas at larger distances it may be marginally stable to the perturbations in the disk plane, having at the same time a high $C_{z} / C_{r}$ ratio, and hence, a large thickness.

NGC 5440. The line-of-sight velocity curves for this galaxy are obtained both for the stars and gas from the [NI] and [O III] lines (Fig. 4). The velocity distribution along the major axis demonstrates a regular nature of the disk rotation, especially for the stellar component, where within the growth segment of the rotation curve the stellar component rotates slower, and velocity varies monotonically, while the velocity of the gas component passes through a peak at the distance of about $10^{\prime \prime}$ from the center. In the plateau segment $\left(r>20^{\prime \prime}\right)$ the velocities of stars and gas even out. The central minimum in the distribution of velocities of gas and stars along the minor axis is an artifact, caused by the slight shift of the slit, which did not pass exactly through the center of rotation. At the presence of a steep velocity gradient, the offset can be as little as $1^{\prime \prime}-2^{\prime \prime}$ to produce it.

The model velocity distributions are compared with the observations in Fig. 5 (right). The rotation curve is determined much more confidently from the stars than from gas: the spread of the gas velocity estimates reaches $50 \mathrm{~km} / \mathrm{s}$ relative to the rotation curve, only slightly decreasing towards the periphery. Surprisingly, noncircular motions of gas is observed in spite of the low intensity of star formation, which could have sustained them.

To build a dynamic model of the galaxy, it is preferable to use the velocity estimates of the stellar component, the radial profile of which has a smooth and symmetrical form. As can be seen from the figure, line-of-sight velocity dispersion of stars exceeds the expected value for the maximal marginally stable disk model by $20-40 \mathrm{~km} / \mathrm{s}$, and this takes place over the entire obtained rotation curve. Therefore, the stellar disk of the galaxy can be considered as moderately overheated. 


\section{DISCUSSION AND CONCLUSIONS}

Out of seven early-type disk galaxies considered in this paper and in [22], the model of a marginally stable disk can explain the observed velocities and velocity dispersions in only two galaxies: NGC 2273 and NGC 3245, and in the inner region of NGC 338 (see Table 2, where the last column contains the indications of an overheated disk ("o" for overheated), or a disk for which the observations are consistent with the hypothesis of its marginally stable state ("m" for marginal)). Both NGC 2273 and NGC 3245 are isolated lenticular galaxies, and the probability of dynamic heating from the environment is minimal, while the remaining galaxies belong to rarefied groups. As for NGC 338, it is not a "classical" lenticular galaxy, since the spiral disk structure is observed there and it is likely to be an early type spiral. Table 2 also lists the mass of the disk and the relative halo mass within four radial scales of the disk. The range of values of the latter is quite typical for disk galaxies.

The results of observations and numerical models of the S0-Sa galaxies confirm the conclusion that a significant proportion of the early-type disk galaxies (but far not all of them) have dynamically overheated stellar disks at the distance of 1-2 radial scales from the center, meaning that their velocity dispersion is significantly higher than that required for the gravitational stability of the disk. Note that the galaxies with overheated disks do not excel neither by the disk mass, nor by the relative mass of the halo, and nor by disk mass-to-luminosity ratio (see Table 2), which argues for the external mechanisms causing the velocity dispersion to increase. Apparently, these galaxies have undergone in the past a stage of mergers with smaller systems, which led to a rapid reduction of the amount of gas in the disk through gas depletion and/or a stimulation of an outburst of activity in the nucleus, able to sweep the gas out of disk. As a number of spectroscopic observations of lenticular galaxies show, their stellar disks are characterized by a high magnesium-to-iron ratio, which makes them similar to the thick disks of spiral galaxies [38, 39]. Then, dynamic heating of the stellar disk would have to take place in the first 1-2 billion years after the trigger of the violent stage of its formation, that is, before the interstellar gas got enriched with iron as a result of activity of the Type I supernovae. In contrast to lenticular galaxies, the galaxies of later morphological types rarely have an excess of stellar velocity dispersion at a distance of several radial scales from the center [12]. Therefore, the early evolution of spiral galaxies generally advanced less vigorously.

A part of lenticular galaxies have avoided intense dynamical heating of the disk. In these cases, the morphological features inherent to this type of galaxies appear to be related to other events, such as a high activity of the nucleus, which may affect the abundance of gas and star formation 
in the galaxy [40], or to the interaction with intergalactic gas, if the galaxy is located in a cluster or a compact group. It is noteworthy that two of the most isolated galaxies contained in our small sample (NGC 2273 and NGC 3245), are precisely the objects where we have reasons to suppose the presence of marginally stable disks. We can therefore conclude that there are at least two different mechanisms, responsible for the formation of lenticular galaxies, and the major part of these galaxies appears to have undergone an early stage of formation of a dynamically "hot" disk.

\section{ACKNOWLEDGMENTS}

The work was supported by the Ministry of Education and Science of the Russian Federation. The results of observations were obtained with the 6-m BTA telescope of the Special Astrophysical Observatory Academy of Sciences, operating with the financial support of the Ministry of Education and Science of Russian Federation (state contracts no. 16.552.11.7028, 16.518.11.7073). The authors are grateful to A. V. Moiseev for the help in conducting observations and valuable suggestions, as well as the referee, who made a number of useful comments. The authors also express appreciation to the Large Telescope Program Committee of the RAS for the possibility of implementing the program of spectroscopic observations at the BTA. We as well made use of the SDSS, supported by the Alfred P. Sloan Foundation, the participating institutions, the National Science Foundation, and the U.S. Department of Energy. This work was supported by the Russian Foundation for Basic Research grants (project nos. 11-02-12247-ofi-m, 12-02-00685), the Presidential Grant no. MD-3288.2012.2, and Dmitry Zimin's non-profit Dynasty Foundation. The authors are grateful to SRCC of the Moscow State University for the use of Chebyshev and Lomonosov supercomputers.

1. E. Noordermeer and J. M. van der Hulst, Monthly Notices Roy. Astronom. Soc. 376, 1480 (2007).

2. A. Boselli and G. Gavazzi, Publ. Astronom. Soc. Pacific 118, 517 (2006).

3. A. Boselli, S. Boissier, L Cortese, and G. Gavazzi, Astron. Nachr. 330, 904 (2009).

4. J. M. Barr, A. G. Bedregal, A. Aragón-Salamanca, et al., Astronom. and Astrophys. 470, 173 (2007).

5. A. Aragón-Salamanca, IAUS 245, 285 (2008).

6. K. Bekki and W. J. Couch, Monthly Notices Roy. Astronom. Soc. 415, 1783 (2011).

7. S. van den Bergh, Astrophys. J. 694, L120 (2009).

8. M. J. Williams, M. Bureau, and M. Cappellari, Monthly Notices Roy. Astronom. Soc. 409, 1330 (2010).

9. O. K. Sil'chenko and I. V. Chilingarian, Astron. Lett. 37, 1 (2011). 
10. E. Noordermeer, M. R. Merrifield, L. Coccato, et al., Monthly Notices Roy. Astronom. Soc. 384, 943, (2008).

11. F. Bournaud, C. J Jog, and F. Combes, Astronom. and Astrophys. 437, 69 (2005).

12. A. V. Zasov, A. V. Khoperskov, and A. S. Saburova, Astron. Lett. 37, 374 (2011).

13. A. Cortesi, M. R. Merrifield, M. Arnaboldi, et al., Monthly Notices Roy. Astronom. Soc. 414, 642 (2011).

14. A. M. Fridman and A. V. Khoperskov, The Physics of Galactic Disks (Fizmatlit, Moscow, 2011) [in Russian].

15. E. Griv and M. Gedalin, Monthly Notices Roy. Astronom. Soc. 422, 600 (2012).

16. A. V. Khoperskov, A. V. Zasov, and N. V. Tyurina, Astron. Rep. 47, 357 (2003).

17. A. V. Zasov, A. V. Khoperskov, and N. V. Tyurina, Astron. Lett. 30, 593 (2004).

18. R. Bottema, Astronom. and Astrophys. 197, 105 (1988).

19. R. Bottema, Astronom. and Astrophys. 275, 16 (1993).

20. A. V. Zasov and A. S. Saburova, Astron. Lett. (in press).

21. A. S. Saburova, Astron. Rep. 55, 409 (2011).

22. A. V. Zasov, A. V. Moiseev, A. V. Khoperskov, and E. A. Sidorova, Astron. Rep. 52, 79 (2008).

23. I. V. Chilingarian, A. P. Novikova, V. Cayatte, et al., Astronom. and Astrophys. 504, 389 (2009).

24. E. Noordermeer, J. M. van der Hulst, R. Sancisi, et al., Astronom. and Astrophys. 442, 137 (2005).

25. E. Noordermeer, J. M. van der Hulst, R. Sancisi, et al., Monthly Notices Roy. Astronom. Soc. 376, 1513 (2007).

26. E. Noordermeer and M. A. W. Verheijen, Monthly Notices Roy. Astronom. Soc. 381, 1463 (2007).

27. L. C. Ho, A. V. Filippenko, W. L. Sargent, and C. Y. Peng, Astrophys. J. Suppl. 112, 391 (1997).

28. J. Mendez-Abreu, J. A. L. Aguerri, E. M. Corsini, and E. Simonneau, Astronom. and Astrophys. 478, 353 (2008).

29. F. Simien and Ph. Prugniel, Astronom. and Astrophys. Suppl. Ser. 131, 287 (1998).

30. V. L. Afanasiev and A. V. Moiseev, Astron. Lett. 31, 194, (2005).

31. I. Y. Katkov and I. V Chilingarian, ASP Conf. Proc. 442, 143 (2011).

32. M. Koleva, Ph. Prugniel, A. Bouchard, and Y. Wu, Astronom. and Astrophys. 501, 1269 (2009).

33. M. Koleva, Ph. Prugniel, and S. De Rijcke, Monthly Notices Roy. Astronom. Soc. 385, 1998 (2008).

34. R. P. van der Marel and M. Franx, Astrophys. J. 407, 525 (1993).

35. D. Le Borgne, B. Rocca-Volmerange, Ph. Prugniel, et al., Astronom. and Astrophys. 425, 881 (2004).

36. M. Cappellari and E. Emsellem, Publ. Astronom. Soc. Pacific 116, 138 (2004).

37. I. Chilingarian, Ph. Prugniel, O. Sil'chenko, and M. Koleva, IAUS 241, 175 (2007).

38. O. Sil'chenko, IAUS 284 (in press); astro-ph:1112.3771.

39. O. K. Sil'chenko, I. S. Proshina, A. P. Shulga, and S. E. Koposov, submitted to Monthly Notices Roy. Astronom. Soc..

40. N. Silk, Astrophys. J. 700, 262 (2009). 